\title{
INTEGRACION DE LA SUPERVISIÓN FINANCIERA EN EL BANCO CENTRAL. LA EXPERIENCIA DE URUGUAY
}

\author{
Edgardo Demaestri* \\ Jorge Ottavianelli** \\ Gustavo Ferro***
}

recibido: marzo 2015 - aceptado: mayo 2015

\begin{abstract}
Resumen
Se analiza la experiencia uruguaya de unificación de la supervisión financiera en un único cuerpo regulatorio dentro del Banco Central. Un regulador integrado puede o no estar situado dentro del Banco Central o ser una institución separada. Si se lo establece en el Banco Central, tiene el potencial de conseguir economías de escala y alcance, mejorar flujos informativos y darle un sesgo prudencial a la regulación. Se analiza la experiencia uruguaya incluyendo la consideración de los pros y contras del proceso. También se estudian las consecuencias de las funciones macro prudenciales para hacer frente a crisis financieras. Por último, se identifican lecciones de esta experiencia.
\end{abstract}

Código JEL: G2, L5

Palabras clave: supervisión financiera integrada; regulación micro-prudencial; regulación macro-prudencial; Uruguay

\footnotetext{
Abstract

We analyze the Uruguayan experience of unifying financial supervision in one regulatory body into the Central Bank in Uruguay. An integrated regulator can

* Banco Interamericano de Desarrollo (BID), edgardodm@iadb.org.

** Facultad de Ciencias Sociales, Universidad de la Republica, Uruguay y consultor privado. Ex Superintendente de Servicios Financieros BCU, jottavianelli@gmail.com

*** Instituto de Economía UADE y CONICET, gferro@uade.edu.ar.
} 
be situated inside the Central Bank, or as a separate institution. Being established inside the Central Bank, it has the potential of achieving economies of scale and scope, improving informative flows, and yielding a prudential bias to regulation. We analyze the experience of the integration of financial regulation and supervision in Uruguay into its Central Bank, including the consideration of the pros and cons of the process. We also study the consequences of macro-prudential functions to face a financial crisis. Finally, we identify lessons arising from this experience.

Key Words: integrated financial supervision; micro-prudential regulation; Uruguay JEL Code: G2, L5

\section{INTRODUCCION}

El objetivo central del trabajo es analizar la reforma hacia un esquema integrado de regulación y supervisión financiera en Uruguay y extraer lecciones de dicha experiencia. El esquema de integración aplicado en Uruguay se llevó adelante unificando la supervisión de todos los servicios financieros en el Banco Central. Ello ha tenido implicaciones tanto en materia de supervisión micro como macro prudencial.

En los últimos veinte años, se observa en muchos países una tendencia a integrar supervisores financieros de diferentes intermediarios y actividades financieras. Considerando que diferentes intermediarios financieros operan en los mismos mercados y compiten por los mismos clientes con productos similares, la tendencia puede parecer a priori como lógica. El sector privado maximiza beneficios en forma integrada de intermediarios diversos y ha tendido a operar conformando conglomerados. En la medida en que el sector privado financiero se conglomera para prestar servicios diversos, la supervisión integrada podría regularlos y supervisarlos más eficazmente.

Sin embargo, hay un amplio debate sobre las ventajas y desventajas de integrar institucionalmente la supervisión financiera. Por un lado, un supervisor integrado, de líneas de negocio diferentes pertenecientes a intermediarios de un mismo grupo, puede promover la transferencia de conocimiento entre sectores, uniformar criterios en áreas donde existen potenciales superposiciones, mitigar el arbitraje regulatorio que pudiera darse en los diferentes mercados con actividades similares pero diferente regulador, y facilitar el óptimo aprovechamiento del capital humano ofreciendo mejores perspectivas de carrera. Se espera que ello baje costos de transacción, genere economías de escala y alcance en la supervisión, y que lleve 
a menores costos administrativos unitarios. Puede aumentar el prestigio y la visibilidad de los supervisores locales a nivel internacional y, al integrar la supervisión, reducir el riesgo de captura por la mayor profesionalización de las autoridades regulatorias. Asimismo, entre los pros de integrar la supervisión financiera, la literatura señala que es una forma de controlar más eficazmente conglomerados financieros dado que, con reguladores múltiples, se podrían realizar arbitrajes regulatorios al maximizar los conglomerados beneficios en forma conjunta mientras persisten zonas grises o vacíos en la regulación y supervisión.

Por otro lado, con la integración se pierde la "competencia entre reguladores" y el proceso mismo de integración presenta problemas de transición al nuevo sistema que merecen especial atención. Un riesgo relacionado, es el del Leviatán regulatorio, es decir, crear una institución demasiado poderosa, autónoma en exceso y difícil de responsabilizar. Requiere adecuados contrapesos al establecer metas precisas, funciones acotadas y rendición de cuentas. Otro riesgo, es la posibilidad que la reforma sea cooptada en su diseño por objetivos subalternos o interesados.

A su vez, el regulador financiero unificado puede o no estar en el banco central. Su presencia allí tiene como principal ventaja la posibilidad de aprovechar sinergias de recursos materiales y humanos, mejorar la fluidez de los flujos informativos y darle un sesgo prudencial a la regulación, fortaleciéndolo para atender los riesgos sistémicos, dado que el banco central es quien en general está a cargo de resolver una eventual crisis bancaria.

Las diferencias nacionales reflejan una variedad de factores que inciden sobre los diseños institucionales, como la evolución histórica, la estructura del sistema financiero, las políticas, las tradiciones, el tamaño del país y de su sistema financiero. La integración de la supervisión financiera comenzó en los países escandinavos, pero el tema ganó el centro del debate en oportunidad de la unificación de supervisores financieros en el Reino Unido en 1997. La reciente crisis financiera internacional en 2008 reactualizó el tema.

La mirada a los casos internacionales denota que la reciente crisis financiera global de 2008 fue de particular gravedad en los dos mercados financieros más complejos y desarrollados del mundo (Estados Unidos y el Reino Unido), donde la forma de estructuración de la regulación y supervisión micro-prudencial era estrictamente opuesta: atomizada y unificada respectivamente. En ambos casos se observaron arbitrajes regulatorios que las autoridades no pudieron detectar a tiempo, o que las tomó enteramente por sorpresa. La reacción en ambos casos 
ha sido aumentar el grado de coordinación de los supervisores y darles mayor poder de intervención. En los dos contextos el banco central resultó fortalecido en sus funciones tras haber efectuado masivas inyecciones de liquidez para ayudar a sanear instituciones en problemas e impedir el colapso de la economía real. En el Reino Unido el supervisor micro-prudencial pasó al Banco de Inglaterra. En Estados Unidos se pasaron funciones micro-prudenciales a la FED en el caso de instituciones sistémicas.

El caso uruguayo muestra similitudes en cuanto a motivaciones con reformas en otros países. Por ejemplo, como en Suecia, el detonante para el fortalecimiento de la supervisión financiera fue una severa crisis sistémica en 2002, que redujo a la mitad la profundidad del sector bancario local en dos años. Salvados los efectos más graves de la crisis y puestos los cimientos para la recuperación del sistema, vinieron los cambios de estructura de supervisión. Estos se montaron sobre la base del supervisor más grande, del segmento financiero más importante del país, se aprovechó el capital humano existente y se puede decir que se produjo sin fricciones. La reforma se realizó en un contexto macroeconómico de expansión y con el crédito recuperándose. En años previos se fortaleció la supervisión de los bancos, intermediarios que son predominantes en el sistema local.

El presente trabajo se divide en secciones, tras esta introducción. La sección I refiere el contexto macroeconómico y del sistema financiero, la sección II presenta la evolución histórica de la regulación financiera en Uruguay, la sección III cuenta la reforma, la sección IV sintetiza la organización resultante, la sección $\mathrm{V}$ discute los aspectos macro prudenciales y la sección VI concluye con las lecciones de la experiencia.

\section{CONTEXTO MACROECONOMICO Y DEL SISTEMA FINANCIERO}

El peso del sector bancario en Uruguay es muy importante, destacándose además que aproximadamente la mitad del sistema financiero por volumen de activos es estatal. La mitad restante son bancos internacionales, no existiendo bancos privados de capital local. El país también se caracteriza en sus intermediarios financieros por la presencia de un pequeño mercado de valores y de seguros, una reforma previsional menos radical que en otros países de la región, la existencia de banca off shore y un sistema financiero bi-monetario.

Uruguay atrajo flujos de capitales en los años 1990s, parte de los cuales fueron originados a raíz de turbulencias macroeconómicas en países vecinos. En 
el año 2000 el sistema bancario uruguayo se posicionaba segundo en la región en términos de profundidad financiera con $51 \%$ del PBI en depósitos, sólo superado por Chile (IMF, 2010).

La crisis financiera de 2002 respondió a múltiples causas, de origen nacional e internacional. Inicialmente la crisis argentina provocó la suspensión de una subsidiaria de un banco que esencialmente tenía depósitos y créditos de titulares argentinos y el cambio de conducta de depositantes no residentes que comenzaron a retirar sus depósitos en búsqueda de instituciones percibidas como más solventes. No hubo inicialmente salida de depósitos del sistema, sino "fly to quality". Debido a un fraude en el principal banco privado del país, ocurrió lo propio con depositantes residentes. La abrupta caída de las reservas internacionales (de algo más de 3,000 a algo más de 500 millones de dólares entre comienzos de 2002 y fines de agosto de ese año), la baja de calificación por parte de agencias crediticias, que llevó el riesgo país de 200 bp a fines de 2001 a casi 3100 bp a fines de julio de 2002, el abandono de la banda de flotación del peso uruguayo, la intervención de otro banco uruguayo de tamaño significativo y la demora de reacción del sistema, pueden anotarse como causales de la corrida y la crisis sistémica de 2002 (López y Ottavianelli, 2003).

Fueron liquidados cuatro bancos, una cooperativa de intermediación financiera y una institución financiera externa, salieron del sistema varias instituciones y otras fueron transformadas y se produjo una reprogramación de los depósitos de los bancos públicos. Ante la falta de liquidez, los bancos debieron liquidar activos y la recesión acentuó la tendencia negativa en la recuperación de los créditos, llevando a las entidades a constituir mayores previsiones (López y Ottavianelli, 2003). Los depósitos de no residentes bajaron un $78 \%$. El crédito cayó a un $24 \%$ del PBI en 2004. Los mayores controles regulatorios redujeron la exposición a préstamos en dólares de los bancos locales y cayó también la exposición del banco público que colocaba en esa época la mayoría de las hipotecas.

Entre fines de 2006 y 2008, el crédito se recuperó. La crisis de 2002-2003 deprimió los niveles de depósitos, pero su recuperación se aceleró en 2007-2008, llegando a tasas de crecimiento del $40 \%$ (en algún trimestre en particular) para mediados de 2008. La expansión crediticia estuvo motorizada por aumentos de los préstamos al consumo y a las empresas. Entre los factores internos que favorecieron esta recuperación del crédito se destaca el hecho de que los bancos limpiaron sus carteras, que se fue recuperando la confianza y retornó la calma, en un contexto en que la economía crecía fuertemente. A nivel internacional, la baja de tasas norteamericana generó nuevamente flujos de capital hacia el país, que 
apreciaron la moneda local. Las políticas macroeconómicas fueron prudentes y a la vez el sector externo tuvo un boom exportador ligado a los buenos precios de las materias primas locales de exportación (Adler et al., 2009).

\section{EVOLUCION HISTORICA DE LA SUPERVISION Y REGULACION FINANCIERA EN URUGUAY}

En sus orígenes, la regulación financiera se reducía a un régimen individual de aprobación legislativa para la autorización de bancos, consagrado en la Constitución de 1830. Con la Ley General de Bancos de 1865 se creó el Comisario de Inspección y Vigilancia bancaria, quien en nombre del Poder Ejecutivo debía controlar la emisión y los balances individuales de los bancos. La regulación bancaria no avanzó durante el siglo XIX porque el Estado era el principal deudor de los bancos y a la vez su regulador. Tras la crisis de 1890, se creó en 1896 el Banco de la República (BROU) en lugar del Banco Nacional, sin atribuciones regulatorias, pero contando con un Departamento de Emisión. Este iría incorporando atribuciones y separándose de la institución hasta convertirse en el antecedente del Banco Central.

En 1916 se creó la Inspección Nacional de Bancos. En 1938 se ampliaron sus cometidos y se trasladó dicha Inspección al Departamento de Emisión. En 1935 se había separado dentro de la estructura del BROU a las funciones bancarias de las de emisión, dotando al Departamento de Emisión de una relativa autonomía en sus funciones. La ley estableció que le competería la supervigilancia y fiscalización sobre la banca, así como ciertas potestades en materia de autorización de nuevas entidades. En 1939 se proveyó al BROU del carácter de Ente Autónomo. Desde los años 1930s hasta 1960 se fueron desplazando facultades desde el Poder Ejecutivo hasta el Departamento de Emisión en términos de supervisión bancaria. En 1960 se transfirieron restantes facultades supervisoras de la Inspección General al Departamento de Emisión y en 1964 se le dotaron de potestades sancionatorias (Gianelli y Abal, 2004).

A fines de 1964 ocurrió una crisis bancaria. Hasta ese momento solo se controlaba la legalidad de las operaciones bancarias, tanto a través de la Tesorería como del Departamento de Emisión del BROU. En los años siguientes, los procesos de supervisión se centraron en la información contable y se dirigieron fundamentalmente a verificar las transacciones (Ottavianelli, 2012). 
La crisis motivó la creación de una red de seguridad (prestamista de última instancia y seguro de depósitos) y una Unidad de Control Permanente dentro del Departamento de Emisión, primero circunscripta a bancos y luego a casas bancarias, cajas populares y entidades de giro financiero. La nueva Constitución de 1966 creó un banco central organizado como ente autónomo, al que se le transfirieron los cometidos y atribuciones del Departamento de Emisión del BROU, confiriendo a la futura legislación el derecho a ampliar o restringir dicho campo. No se dictaría una Carta Orgánica hasta 1995, sino que se acumularon disposiciones y reglamentaciones que conformaban una "carta orgánica invisible" (Gianelli y Abal, 2004).

Producto de la crisis de 1982, se produjeron una serie de cambios en los procesos de supervisión y regulación. En 1986 se estableció el Plan de Cuentas obligatorio para todas las instituciones, haciendo comparable la información. En 1989, siguiendo la tendencia internacional del Comité de Basilea, se dictaron una serie de normas para acotar los riesgos de los bancos: 1) la norma de evaluación y clasificación de riesgos estableció cuatro categorías de riesgo y determinó criterios objetivos y subjetivos para la inclusión en cada una de ellas a los deudores; 2) se fijaron requerimientos de capital según la composición de los activos; 3) se establecieron topes de riesgos por préstamos al sector no financiero; 4) se definieron relaciones técnicas con límites a los activos inmovilizados, tenencia de valores públicos y radicación de activos en el país; 5) se reguló la exigencia de una mayor participación de los auditores externos requiriéndoles nuevos informes.

En 1991 se incorporó en el proceso de supervisión in situ la metodología de calificación de entidades denominada PADUL, que es el correlato local de las calificaciones CAMEL en Estados Unidos. Estos ratings analizan el capital, los activos, la dirección y administración, las utilidades y la liquidez de los bancos. El método califica de 1 (sin problemas) a 5 (con necesidad de ayuda externa) a las entidades. En 1993 se estableció una nueva normativa de carpeta de clientes (Comunicación 93/60) donde se establecieron requisitos para que los bancos evaluaran los riesgos de crédito. Ese año se creó la Superintendencia de Instituciones de Intermediación Financiera, ratificada por Ley dos años después, confiriéndole autonomía técnica dentro del BCU.

Entre 1993 y 1996 el BCU sumó a sus funciones de política monetaria, supervisión bancaria, asesor, banquero, representante financiero del gobierno y administrador de las reservas, potestades regulatorias adicionales en cuatro mercados (seguros, AFAP, valores y fondos de inversión), abarcando la totalidad del sistema financiero (Gianelli y Abal, 2004). 
La crisis de 2002 reveló limitantes del modelo supervisor en vigencia. La clasificación PADUL aplica los mismos procedimientos para todas las instituciones, con independencia del perfil de riesgos de la entidad analizada. La mayor parte del análisis se vuelca a evaluar el desempeño histórico, motivando que la supervisión sea reactiva. El análisis es mayormente cuantitativo, no contemplando detalles de calidad del negocio, de los accionistas y del gobierno corporativo de la entidad. No hay evaluación de riesgos operacionales ni de su administración, sus procesos de control interno, ni de las funciones del auditor interno (véase López y Ottavianelli, 2003, para un abordaje conceptual y Bergara et al., 2001 para un análisis empírico).

Las medidas regulatorias que se introdujeron después de la crisis de 2002, incluyeron una más detallada clasificación y previsionamiento de préstamos, requisitos separados de liquidez, reglas de maduración y calce de monedas, límites a la exposición individual, sectorial y por país, exigencias de prácticas de manejo internas de los riesgos para los bancos, periódicos tests de stress de deudores corporativos, un bureau de crédito nacional preexistente que consolida información nacional sobre riesgo de los deudores, medidas administrativas sobre bancos que no cumplan, como requerimientos adicionales de capital o prohibiciones de expansión de préstamos (Adler et al., 2009, Wezel, 2010).

\section{LA REFORMA}

La crisis de 2002 fue el disparador para todas las reformas. A fines de 2002 ya circulaban borradores y se inició el proceso de cambios. El Poder Ejecutivo asumido en el 2005 propuso al Parlamento una nueva Carta Orgánica para el BCU, oportunidad en que fue revisado el modelo de supervisión. En octubre de 2008, el Parlamento aprobó la Ley 18.401 que reformó la Carta Orgánica del Banco Central del Uruguay (BCU). Por dicha ley, se unificó la regulación y supervisión de todos los participantes del sistema financiero, al crearse la Superintendencia de Servicios Financieros (SSF) como una dependencia del BCU.

De acuerdo con este ordenamiento jurídico, las tareas de supervisión sobre el sistema financiero las desarrolla el BCU (a cargo del sistema de pagos y la política monetaria) a través de la SSF, y existen comités para coordinar aspectos de política monetaria y supervisión financiera con el Ministerio de Economía y Finanzas (MEF). La Corporación de Protección del Ahorro Bancario (COPAB) es la entidad encargada de administrar el seguro de depósitos. Las relaciones entre la COPAB y la SSF están amparadas por Memorándums de Entendimiento. 
El debate, en aquella oportunidad a nivel del BCU y el MEF, analizó los pros y contras de dejar inicialmente la supervisión bajo la órbita del Banco Central y luego de integrar a otros intermediarios no bancarios. Los principales argumentos evaluados fueron la necesidad de mitigar y en su caso explicitar conflictos de interés y de maximizar las economías de escala en el manejo de los recursos.

El primer elemento se relaciona al hecho de que antes de la reforma el BCU tenía a su cargo las tareas de prestamista de última instancia, de regulador prudencial y, desde 2005, de proveedor del seguro de depósitos. Un banco central que provee el seguro de depósitos tiene conflictos de interés: maximizar la prestación del seguro alivia su rol de prestamista de última instancia, lo cual a su vez aumenta el riesgo moral de los depositantes. Se zanjó la situación separando del BCU el rol de asegurador de los depósitos, que fue conferido a la COPAB, mientras que la función de regulación y supervisión macro-prudencial permaneció a cargo del BCU. Asimismo, la nueva Superintendencia se diseñó con el máximo grado de desconcentración en el $\mathrm{BCU}$, a los efectos de mitigar los potenciales conflictos entre la regulación y supervisión por un lado, y la política monetaria y el papel de prestamista de última instancia por otro. Es de destacar que el BCU tiene una historia de profesionalidad y respeto social.

La posible existencia de economías de escala merece especial importancia en el caso de un país de las dimensiones de Uruguay, con población cercana a los países nórdicos o bálticos que han unificado sus sistemas (argumento de los sistemas financieros pequeños de Taylor y Fleming). Aquí el concepto puede analizarse desde dos puntos de vista. El aumento de escala podría llevar a una ganancia en eficiencia al lograr realizar la misma producción con menores costos, o bien la mejora podría implicar no gastar menores recursos pero sí lograr incrementos en la calidad y en la cantidad de productos (servicios) generados. Este parece ser el caso en Uruguay, donde se aprecian mejoras en servicios de regulación y supervisión, a partir de haber unificado procesos regulatorios y de supervisión, sobre la base del enfoque de riesgos. Este enfoque supone como principio que a igual tipo de riesgo, se aplicará igual regulación y supervisión, más allá de la entidad que se trate.

El proceso productivo de la regulación y supervisión financiera tiene como insumos básicamente al capital humano e información. El capital humano en una institución de supervisión podría agruparse en tres conjuntos: el que poseen quiénes toman las decisiones en primer término (combinando conocimientos de gerencia, negociación y técnicos, comprende a directivos y la línea gerencial), quiénes realizan labores técnicas en segundo lugar (jurídicas, contables, económicas, de administración, operativas, financieras), y finalmente quiénes aportan servicios 
(administrativos internos, informáticos, mantenimiento, etc.). En los dos primeros casos se puede ahorrar recursos: las tareas directivas se pueden comprimir en un directorio unificado, contra varios directorios; lo mismo ocurre para las gerencias. En los estamentos técnicos no hay una relación tan directa, pero sí parece ser que la unificación permite una interacción entre expertos de distintas áreas, con conocimientos muchas veces complementarios y trabajos que se desarrollan en forma común y concentrada. En los casos de servicios, está claro el potencial de ahorro de recursos. Centralizando directorios y oficinas técnicas se pueden ahorrar funcionarios de servicios diversos.

Respecto del otro insumo, la información, se incurre en varios costos: los de pedir (internamente), los de procesar lo recibido y la carga sobre los intermediarios que deben proveer. El tercer punto no recae directamente sobre la institución, pero incide en los costos de las entidades y en mayor o menor medida en la eficiencia del sistema y en los costos de intermediación que en parte terminarán afrontando los consumidores de los servicios. El volumen de información pedido tiene que ver con los recursos internos afectados a su procesamiento, en términos de capital humano y sistemas. La fusión de supervisores es una oportunidad para migrar a procesos y sistemas únicos, formatos comunes de información y que haya una ventanilla o conducto informativo exclusivo que centralice la vinculación informativa entre el supervisor y los supervisados.

Desde el inicio del proceso de integración de la regulación y supervisión financiera se ha verificado la existencia de economías de escala tanto en la oferta de información (por ejemplo, desde el 2011 se realiza un reporte de estabilidad financiera que abarca a todos los sectores y reemplaza a varios informes), como en la demanda de la misma (el criterio de ventanilla única vigente permite reducir la información solicitada a las instituciones lo que implica una reducción en los costos administrativos para el supervisor y de la "carga excedente", para las entidades).

El BCU es un ente creado por la Constitución de la República y, según su Carta Orgánica ${ }^{1}$, tiene plena autonomía técnica, administrativa y financiera. Sus funciones primordiales son la "estabilidad de precios que contribuya con los objetivos de crecimiento y empleo", y "la regulación del funcionamiento y la supervisión del sistema de pagos y del sistema financiero, promoviendo su solidez, solvencia, eficiencia y desarrollo". Para cumplir con dichas finalidades tiene la atribución de exclusividad de emisión, de aplicación de instrumentos monetarios,

1 Texto Ordenado aprobado por Ley 16696/95, con las modificaciones introducidas por las leyes 18401/08, 18643/10 y 18670/10. Consta de trece capítulos divididos en sesenta artículos. 
cambiarios y crediticios, es asesor económico, banquero y representante financiero del Estado, administra las reservas internacionales y es banquero de intermediarios financieros. Representa al gobierno ante organizaciones multilaterales de crédito, y regula y supervisa normativamente las entidades públicas y privadas que integran el sistema financiero.

La autoridad ejecutiva de la Superintendencia de Servicios Financieros recae en un funcionario designado y cesado por unanimidad por el Directorio del BCU por un período de ocho años, que actuará con desconcentración y autonomía técnica y operativa. El Directorio puede avocarse (es decir, atraer a sí la resolución de un asunto o causa cuya decisión correspondería a un órgano inferior) en cualquier momento respecto de las normas dictadas por la Superintendencia, revocar de oficio y modificar actos administrativos de aquélla. La Superintendencia es el vehículo a través del cual el BCU ejerce la regulación y fiscalización de cualquier entidad integrante del sistema financiero.

Corresponde a la Superintendencia dictar normas generales e instrucciones particulares de prudencia, para promover la estabilidad, solvencia, transparencia y el funcionamiento ordenado y competitivo de las entidades supervisadas y los mercados en que actúan, proteger a los consumidores de servicios financieros, prevenir y controlar el lavado de activos y el financiamiento del terrorismo. Le corresponde habilitar las entidades supervisadas una vez autorizadas por el Poder Ejecutivo, autorizar la apertura de dependencias de las mismas, emitir opinión o decidir sobre proyectos de fusiones, absorciones y transformaciones, autorizar la emisión y transferencia de acciones de entidades que sean sociedades anónimas, aprobar planes de recomposición o adecuación patrimonial.

La Superintendencia puede requerir información, exhibición de registros y documentos, establecer el régimen informativo contable de las entidades, reglamentar su publicación, evaluar periódicamente la situación económico-financiera de las entidades, el cumplimiento de las normas vigentes y la calidad de su gestión. Puede aplicar sanciones (observaciones, apercibimientos y multas), proponer sanciones graves al Directorio (fijar multas mayores al 10\% de la responsabilidad patrimonial básica), intervención, suspensión o revocación de autorización o habilitación. También puede instruir sumarios al personal superior, otorgar la no objeción en caso necesario a designaciones de personal superior de entidades supervisadas, requerir reestructuras de organización, controlar consolidadamente actividades en el país y en el exterior de las entidades supervisadas. Debe llevar registros, acordar bases de entendimientos con la COPAB, y tiene atribuciones de organización interna. 
Dentro del BCU existe un Comité de Regulación y Supervisión integrado por dos miembros del Directorio, el Superintendente de Servicios Financieros y otros dos funcionarios designados por el Directorio para brindar el asesoramiento que requiera el Directorio para tomar las decisiones que correspondan en materia de regulación y supervisión, analizar y asesorar al Directorio sobre las propuestas de políticas y planes preparados por la Superintendencia, monitorear la aplicación de las políticas establecidas y el desarrollo de los planes de trabajo aprobados y opinar sobre las propuestas de Memoria y Plan de Actividades.

\section{LA ORGANIZACION RESULTANTE}

En la concepción de las autoridades que ejecutaron la reforma predominó una visión de economía institucional. Se compara el problema de agencia al de una firma, donde en este caso los accionistas son la sociedad toda y el agente la SSF. Las empresas privadas han intentado resolver los problemas de delegación creando un directorio como cuerpo representativo de los intereses de los accionistas que define las políticas y controla la ejecución. En organismos públicos los problemas de agencia están aún más presentes: los ciudadanos se expresan en ocasiones espaciadas en el tiempo cuando votan, y sin un directorio, un superintendente puede ser todopoderoso por un lado, pero muy frágil por el otro y actuar como un fusible ante shocks.

Un directorio cumple el doble rol de actuar como paragolpes ante la política (confiriéndole la necesaria continuidad al trabajo técnico), a la vez que el superintendente debe rendir cuentas ante dicho directorio, que tiene responsabilidad frente a la ciudadanía. En ese sentido, la organización local ha descansado en un superintendente técnico, con estabilidad y autonomía, un mandato solapado entre dos períodos presidenciales (en Uruguay son de cinco años sin reelección, y el superintendente dura ocho años en funciones), cumpliendo un rol de "gerente general" y un directorio que lo controla. El presupuesto de la SSF proviene de fondos del BCU. A su vez, existe una tasa sobre los supervisados (no están incluidos los agentes de bolsa) de donde proviene aproximadamente un $80 \%$ de los fondos presupuestales.

De la Superintendencia dependen dos Intendencias (Supervisión y Regulación), la Gerencia de Administración y Sistemas, la Unidad de Información y Análisis Financiero (UIAF), y el Departamento de Métodos. 
Las intendencias se dividen en gerencias, éstas en departamentos, que a su vez contienen unidades, de las cuales dependen los analistas. Los funcionarios de la SSF son funcionarios de carrera. La SSF presenta una pirámide muy jerarquizada con cinco rangos directivos (Superintendente, Intendente, Gerentes, Jefe de Departamento y Jefe de Unidad) y cinco niveles de analistas (I, II, III, IV y V). Un analista del máximo nivel (I) tiene remuneración neta cercana a la mitad de la que percibe el superintendente. En tanto un analista de mínimo nivel (V) percibe una sexta parte del salario neto del superintendente. Los cargos de analistas de nivel IV y V son los que tienen una rotación potencial mayor, dado que los ascensos son lentos y el sector privado puede ser una alternativa atractiva para esos recursos humanos.

La Intendencia de Regulación Financiera se compone de cuatro Departamentos: Autorizaciones y Registros, Normas de Regulación Financiera (servicio que propone las normas y a quien cabe resolver los arbitrajes regulatorios), Información y Atención a Usuarios del Sistema (tiene a su cargo las funciones de Defensa del Consumidor), Estudios de Regulación Financiera (donde se elaboran las estadísticas, se realizan estudios y se sitúa la función de Defensa de la Competencia). Todos los departamentos tienen alcance global (sobre todos los sectores supervisados).

La Intendencia de Supervisión Financiera se compone de tres gerencias: Supervisión de Empresas de Intermediación Financiera, Supervisión de Riesgos y Supervisión de Seguros, AFAP y Mercados de Valores. Las intervenciones son de tipo matricial. Hay especialistas sectoriales pero también visiones transversales de Supervisión de Riesgos en todo el sistema (tanto de mercado como de crédito y operativo). La Gerencia de Supervisión de Empresas de Intermediación Financiera tiene dos departamentos: uno que tiene a cargo la Supervisión de Bancos (12 entidades) y otro que se encarga de la Supervisión de Empresas no Bancarias (IFES - off shore, captando y prestando sólo a no residentes- Casas Financieras - parcialmente off shore, que no capta de residentes pero presta a residentes y no residentes-, cooperativas de intermediación financiera, administradoras de círculos de ahorro previo y administradoras de crédito). Por su parte, la Gerencia de Supervisión de Seguros, AFAP y Mercado de Valores se divide en un Departamento de Seguros y otro de Mercado de Valores (49 corredores, 2 Bolsas, 1 AFISA) y control de 4 AFAP..

La UIAF tiene una Unidad de Información y Análisis (que realiza las labores correspondientes a inteligencia financiera), una Unidad de Casas de Cambio, y 
una Unidad de Control de Lavado de Activos que analiza el riesgo de Lavado de Activos y Financiamiento del Terrorismo de forma transversal en todo el sistema.

La Gerencia de Administración y Sistemas es la ventanilla única (principalmente on-line, habiéndose reemplazado actividades que se hacían previamente en papel), tanto para la entrada como para la salida de información, denuncias, quejas por parte de las entidades, mientras que la relación con los usuarios se vehiculiza a través del Departamento de Atención al Usuario.

Por último, el Departamento de Métodos tiene a su cargo la elaboración y el seguimiento del cumplimiento del Plan Estratégico de la SSF y de los Procesos (con mejoras que alcanzan a toda la Superintendencia). El Plan Estratégico de la SSF forma parte del Plan Estratégico del BCU. La unificación de Procesos permitió alcanzar homogeneidad en los mismos, aprendizaje y economías de escala.

Un enfoque común de riesgos se desarrolló desde el inicio de la nueva Superintendencia, pero a partir del año 2011 se realizó la revisión de toda la normativa en un proceso que en primera instancia uniformiza los formatos por tema de cada recopilación de normas y elabora un cronograma para la eliminación de los potenciales arbitrajes regulatorios. Esta eliminación se concreta en una segunda etapa. Asimismo, se diseña un proceso de supervisión que abarque todos los sectores con el criterio sustentado anteriormente: a igual tipo de riesgo, igual proceso de supervisión.

Previo al proceso de integración, los conglomerados financieros (que de por sí no son tan importantes en Uruguay como en países desarrollados), no eran supervisados como tales, sino que había "miradas" independientes y especializadas sobre cada intermediario. Actualmente, la SSF continúa trabajando a fin de mejorar la supervisión de los conglomerados financieros. En este sentido, como primer paso se procedió a definirlos con precisión. Se reconocen dos tipos de conglomerados financieros: aquellos que jurídicamente operan como tales y otros donde hay accionistas comunes pero no se consolidan. Lo sustancial es supervisarlos de la misma manera. Se han definido los diferentes conglomerados con sus integrantes y se han realizado informes sobre la base de la información recibida, prestando especial atención a las operaciones intra-grupo y al Gobierno Corporativo del conglomerado. Para esto se ha creado una unidad independiente que realiza este seguimiento. 
Aquí el énfasis son las funciones integradas: se integra la regulación, la atención al usuario, la supervisión, la defensa de la competencia y las autorizaciones. Se buscan análisis globales con procesos unificados.

\section{INTEGRACION EN EL BANCO CENTRAL: ASPECTOS MACRO-PRUDENCIALES}

Junto con el cambio institucional, se efectuaron cambios en la regulación macro-prudencial que buscan darle a la banca local las fortalezas que no tenía en 2002.

A continuación se analizarán dichos cambios en dos vertientes: la primera referida a las instituciones (comités en el que participan miembros de las entidades con responsabilidades micro-prudenciales, de política monetaria y fiscales), y la segunda vinculada con los instrumentos macro-prudenciales que se han introducido para hacer más seguro al sistema.

La Carta Orgánica creó tres comités: el de Politica Monetaria (COPOM, Artículo 31 de la Carta Orgánica), el de Regulación y Supervisión (Artículo 39 de la Carta Orgánica), el cual cruza a los responsables de cada área, y el Comité de Coordinación Macroeconómica (Artículo 41 de la Carta Orgánica), que vincula al Banco Central con el Ministerio de Economía. A los anteriores se agregó por Decreto un cuarto Comité de Estabilidad Financiera.

1) En el COPOM participa el Directorio del BCU, el Superintendente de la SSF y los Gerentes del BCU a cargo del diseño de la política monetaria y los mercados;

2) Dentro del BCU existe un Comité de Regulación y Supervisión integrado por dos miembros del Directorio, el Superintendente de Servicios Financieros y otros dos funcionarios designados por el Directorio para brindar el asesoramiento que requiera el Directorio para tomar las decisiones que correspondan en materia de regulación y supervisión, analizar y asesorar al Directorio sobre las propuestas de políticas y planes preparados por la Superintendencia, monitorear la aplicación de las políticas establecidas y el desarrollo de los planes de trabajo aprobados y opinar sobre las propuestas de Memoria y Plan de Actividades. Asisten, los Gerentes a cargo de la política monetaria y los mercados.

3) En lo que respecta a las relaciones con el Poder Ejecutivo y el Legislativo, hay un Comité de Coordinación Macroeconómica integrado por el Ministro 
de Economía y Finanzas, dos funcionarios de su cartera que aquél designe y los tres Directores del BCU. Tiene la función de la puesta en común de información relacionada con las competencias propias de un banco central (política monetaria) y la política económica general, el establecimiento de la meta de estabilidad de precios comprometida y el régimen cambiario. En caso de empate, se estará a lo que resuelva el Poder Ejecutivo.

4) Se creó por decreto un Comité de Estabilidad Financiera integrado por el Ministro, el Presidente del BCU, el Superintendente y el Presidente de la COPAB. Entre sus consideraciones, se caracteriza la estabilidad financiera como un bien público, y se adjudica a este Comité el cometido de intercambiar información y visiones para la supervisión macro-prudencial, promover estudios para identificar y medir riesgos sistémicos, elaborar planes de contingencia y alerta temprana en situaciones previas a crisis y propiciar la coordinación con instituciones regionales e internacionales en temas vinculados a la estabilidad financiera.

La integración cruzada de los dos primeros comités (Política Monetaria y Regulación y Supervisión) busca un objetivo de compartir información y otro de sensibilización de las partes al mejorar el entendimiento de los problemas de la otra parte y las interacciones, así como ganar en sinergias. Nuevamente, los potenciales conflictos de interés persisten, pero se explicitan. Hay mayor coordinación y conocimiento de los problemas y posiciones de cada parte. Al tercer comité (Coordinación Macroeconómica) cabe la puesta en común de información y el establecimiento de la meta de inflación. El cuarto comité (Estabilidad Financiera) enfatiza los aspectos macro-prudenciales y hace participar al Tesoro en el análisis previo a eventuales crisis y, una vez ocurridas, lo coloca como responsable de instrumentar parcialmente el salvataje necesario con dinero de los contribuyentes.

Con respecto a los instrumentos macro-prudenciales que se están utilizando en Uruguay, aunque guardan similitudes con medidas similares introducidas en otros países, algunas cuestiones responden a las particularidades de la economía local, en particular su condición de bimonetaria, y un punto sobre liquidez combina imaginativamente un instrumento de política monetaria con un criterio prudencial.

1) Los requerimientos de capital van en camino a alinearse a Basilea III, pero a nivel macro-prudencial se modifican los mismos por tamaño (se recordará que tamaño, junto con apalancamiento e interconexión son los tres rasgos que permiten caracterizar a una institución como potencial generadora de riesgo sistémico en la lógica de Basilea III), en proporción a la custodia de valores que se mantienen y tiene un componente idiosincrásico de Uruguay, referido a su condición bimonetaria, por posición de cambio. 
2) Previsionamiento dinámico de pérdidas por préstamos. El instrumento busca cumplir un rol asimilable a los estabilizadores automáticos en la política macroeconómica anticíclica. Las previsiones son una forma de cobertura contable para futuras pérdidas. Se sabe que en el momento malo del ciclo los préstamos incobrables aumentarán, en tanto que es menor su incidencia en épocas de auge macroeconómico. Entonces, los requerimientos, mediante fórmula, obligan a acumular previsiones en las épocas buenas y desacumularlas en las épocas malas, de modo de absorber más favorablemente los shocks. El sistema ha sido ponderado desde el punto de vista filosófico, aunque se ha apuntado desde la industria a que por características de la fórmula tiende a la sobreacumulación de previsiones en épocas buenas y a un uso de ellas en las malas que es más lento que la evolución del ciclo. Recientemente se ha modificado la fórmula procurando atender dichas observaciones, habiendo sido ajustado estudiando los potenciales ciclos y el acompañamiento de las previsiones al ciclo. El previsionamiento dinámico data de 2001, y por tanto el fondo se creó, y se usó en seguida. No se ha utilizado luego de la crisis pues el ciclo económico ha sido en alza desde esa fecha.

3) Requisitos de reservas. Separa los encajes (instrumento de política monetaria) de los requisitos de liquidez (concepto prudencial más amplio). Los bancos deben cumplir con el mayor de ambos. En Uruguay, a la visión tradicional de encaje como instrumento monetario, se cruza una visión prudencial de liquidez. Los requisitos de liquidez se fundan en la percepción del supervisor prudencial respecto al grado en que los retiros de depósitos pueden superar lo rutinario. Se constituyen en épocas buenas para usarse en épocas de tensión. La regulación ordena que se cumpla con aquellos requisitos de liquidez o con los encajes, según sea el mayor. Los encajes se constituyen en el BCU y no se remuneraron hasta fecha reciente. En cambio los requisitos de liquidez pueden constituirse en instrumentos líquidos de buena calidad, en el país o en el extranjero, que son por definición remunerados. Eso reduce la carga para los bancos de tener inmovilizados esos recursos. Desde la industria se ha observado que una forma alternativa de atender a este propósito sería la existencia de una ventanilla de descuento del BCU que tuviera dos líneas: una de liquidez, permanente, y otra para atender situaciones críticas. Por la historia de las crisis financieras, en tanto, la percepción imperante es que recurrir a la ventanilla en condiciones normales llama la atención del mercado y siembra dudas sobre la entidad que pide asistencia, por lo cual, los bancos procurarían no utilizarla. A partir de la crisis financiera, se ha prestado atención especial a los depósitos de no residentes, sobre los que, luego 
de un análisis sistémico, se ha decidido mantener un requisito de liquidez del 30\%, que es el más alto del sistema.

4) Límites a los préstamos y distinta ponderación para previsionar los créditos personales por moneda. Si bien hay límites a la exposición por empresa, también existen límites por país, según el grado de calificación de riesgo del mismo y límites al sector público. Asimismo, a los efectos de constituir previsiones por cada crédito, se deben realizar pruebas de stress sobre los créditos con respecto a la variación del tipo de cambio según dos escalones definidos. Si el flujo de caja se ve afectado por la variación del tipo de cambio, se deben constituir previsiones para esos créditos. El descalce de los sectores económicos se estudia a través de indicadores, pero no se regula. En una economía bimonetaria, los peligros a la estabilidad financiera que representa la exposición de los sectores transables y no transables a créditos en moneda extranjera, son muy distintos. Un exportador, en el extremo, tiene flujos de caja positivos en moneda extranjera, de modo que está calzado si se endeuda en divisas. Pero un asalariado del sector servicios o una empresa del sector no transable con clientes en el mercado local, por pensar un par de ejemplos, tienen ingresos en pesos uruguayos y por ende quedan descalzados si teniendo deudas en dólares se producen movimientos de alguna importancia en el mercado cambiario. El BCU lleva un indicador de descalce de monedas que permite clasificar a las personas y empresas según la calidad de transable internacionalmente de su producción, a partir de la moneda de sus flujos de ingreso. Los bancos deben efectuar más aportes de capital por los préstamos otorgados en moneda extranjera (pero esto está reseñado en el punto siguiente). Las previsiones para pérdidas esperadas se sensibilizan a una banda de variación del tipo de cambio real.

5) Relación entre el préstamo y valor del activo. Su lógica obedece a acotar la posibilidad de burbujas en los precios de los activos. Todos los créditos del sector privado tienen ponderación distinta para el cálculo de capital según estén otorgados en moneda nacional (100\%) o en moneda extranjera (125\%).

\section{CONCLUSIONES}

Entre las lecciones extraídas de la experiencia se pueden enumerar:

1) Las consideraciones de escala tienen mucha relevancia por la dimensión del país. El país es comparable por su población a Noruega, Finlandia o Dinamarca, o los estados Bálticos. Aquí se ha enfatizado que a los posibles 
ahorros puntuales de recursos presupuestarios (por personal y plataformas informáticas) se deben adicionar en la consideración las ganancias en calidad y cantidad de información producida para tomar decisiones regulatorias (aumentando los estándares para intermediarios financieros no bancarios) y posiblemente una baja de la "carga excedente" (costos de cumplimiento) sobre las firmas financieras por la existencia de una ventanilla única. También se ha migrado a sistemas únicos y ha mudado la forma en que se colecta la información, desde soporte papel a soporte informático.

2) La hegemonía bancaria dentro de los intermediarios financieros explica la centralización de la supervisión bajo la jurisdicción del banco central. La separación del seguro de depósitos parece un contrapeso, dados los argumentos contrapuestos en relación con la explicitación de los conflictos de interés entre el supervisor y el asegurador de depósitos que se han sugerido al discutir las funciones macro-prudenciales y micro-prudenciales del banco central. Este tiene las manos libres para ser exigente con las entidades, por una parte, y para hacer frente a turbulencias macroeconómicas con la política monetaria, a la vez que se lo descarga de presiones (por lo menos a nivel de una crisis limitada y como señal al público) al encargar el rol de asegurador de los depósitos a otro organismo.

3) La industria bancaria local es mitad estatal y mitad transnacional. Esta última parte tiene como actores a bancos diversificados en conglomerados que abarcan las otras actividades reguladas. Por el lado estatal, hay una suerte de conglomerado implícito que abarca la mitad del sistema, pero en general no existen signos de que actúe como tal.

4) Se espera que la integración eleve los estándares de la supervisión de otros intermediarios con desarrollo más modesto en el país a los niveles de la banca. Ello puede implicar el fortalecimiento de los recursos humanos allí destinados, y quizás tensión entre culturas y prácticas en la transición.

5) Un riesgo del nuevo esquema es que el supervisor unificado se yerga en el demandante monopsonista del capital humano en el sector financiero nacional. Desde el punto de vista de la eficacia en la prestación del servicio, a priori formar un "hub" donde interactúe dicho capital humano y se comparta formación y experiencia es positivo. Pero los incentivos de carrera de los funcionarios tienden a acotarse cuando no hay competencia por sus saberes, el empleo es de por vida y hay escasa rotación. El supervisor unificado, cuya función no es modificar los incentivos en todo el mercado laboral local, pero sí cuidar que su equipo humano sea profesional y sus calificaciones y capacidad de respuesta estén al día, debe mirar con imaginación formas de proveer carrera y desafíos profesionales a los funcionarios. 


\section{BIBLIOGRAFIA}

Adler, G., M. Mansilla \& T. Wezel (2009). Modernizing Bank Regulation in Support of Financial Deepening: The Case of Uruguay. Monetary and Financial Systems Department, International Monetary Fund, IMF Working Paper WP/09/199.

Aizenman, J. (2010). Macro prudential supervision in the open economy, and the role of central banks in Emerging Markets. Open Economies Review, 21, 465-482.

Bergara, M. \& J. Licandro (2002). Regulación prudencial y ciclos de crédito: un enfoque microeconómico. Monetaria, XXV (2), 141-160.

Bergara, M., Licandro G., \& Ottavianelli, J. (2001). Un enfoque complementario para la supervisión bancaria en Uruguay. Revista de Economía, segunda época, $\operatorname{VIII}(1), 5-30$.

Brost, H., H. Loechel \& H. Xianz Li. (2008). Benefits and Costs of Integrated Financial Services Providers (IFSP) - State-of-the-Art in Research. Frankfurt School on Finance and Management, EU-China BMT Working Paper Series 006.

Carmassi, J. \& R. Herring R. (2008). The Structure of Cross-Sector Financial Supervision. Financial Markets, Institutions \& Instruments, 17(1), 51-76.

Banco Central del Uruguay (s.f.). Texto ordenado de la Carta orgánica. Recuperado de http://www.bcu.gub.uy/Acerca-de-BCU/Normativa/Paginas/Default.aspx

Chan-Lau, J. (2010). Regulatory Capital Charges for Too-Connected-to-Fail Institutions Practical Proposal. International Monetary Fund. Working Paper No. 10/98.

Recuperado de http://www.imf.org/external/pubs/ft/wp/2010/wp1098.pdf

Constitución de la República Oriental del Uruguay (1997). [en línea]. Recuperado de http://www.rau.edu.uy/uruguay/const97-1.6.htm

Banco Central del Uruguay (s.f.). Texto ordenado de la Carta orgánica. Recuperado de http://www.bcu.gub.uy/Acerca-de-BCU/Normativa/Paginas/Default.aspx

De Luna Martínez, J. \& Rose T. (2003). International Survey of Integrated Financial Sector Supervision. Financial Sector Operations and Policy Department, World Bank, Policy Research Working Paper 3096,

Di Giorgio, G. \& C. Di Noia C. (2008). Designing a Regulatory and Supervisory Framework for Integrated Financial Markets Capítulo 10. En A. Zazzaro, M. Frattiani, \& P. Alessandrini (Eds.), The Changing Geography of Banking and Finance. (pp. 215-232). doi:10.1007/978-0-387-98078-2_10

Entrevista con Julio De Drun (Presidente Asociación de Bancos, 19/12/11).

Gianelli, D. \& M. Abal (2004). El diseño institucional de la regulación y supervisión financiera en el Uruguay. Revista de Economía, Segunda Época XI (1), 5-72. 
Hanson, S., Kahsyap, A. \& Stein, J. (2011). A Macroprudential Approach to Financial Regulation. Journal of Economic Perspectives, 25 (1), 3-28.

HM Treasury (2010). A new approach to financial regulation: judgement, focus and stability

Recuperado de https://www.gov.uk/government/uploads/system/uploads/ attachment_data/file/81389/consult_financial_regulation_condoc.pdf

International Monetary Fund. (2010). IMF Country Report No. 10/48

Laeven, L. \& Valencia, F. (2010). Resolution of Banking Crises: The Good, the Bad, and the Ugly. International Monetary Fund, Working Paper WP/10/146.

López, N. \& Ottavianelli, J. (2003). La crisis financiera del 2002 y las oportunidades para la supervisión. Revista de Economía, Segunda Época, $(X), 2$, 222-252.

Otavianelli, J. (2012). El gobierno corporativo en los bancos: las crisis, sus propietarios y la regulación. (Tesis Doctoral en Finanzas), Universidad del CEMA.

Seelig, S. \& Novoa, A. (2009). Governance Practices at Financial Regulatory and Supervisory Agencies. Monetary and Capital Markets Department, International Monetary Fund, IMF Working Paper 09/135.

Wezel, T. (2010). Dynamic Loan Loss Provisions in Uruguay: Properties, Shock Absorption Capacity and Simulations Using Alternative Formulas. International Monetary Fund, Working Paper WP/10/125.

Winkler, A. (2010). The Financial Crisis: A Wake-Up Call for Strengthening Regional Monitoring of Financial Markets and Regional Coordination of Financial Sector Policies? Asian Development Bank Institute, Working Paper Series 199.

(C) 2015 por los autores; licencia otorgada a la Revista Estudios Económicos. Este artículo es de acceso abierto y distribuido bajo los términos y condiciones de una licencia Atribución-No Comercial 3.0 Unported (CC BY-NC 3.0) de Creative Commons. Para ver una copia de esta licencia, visite http://creativecommons.org/ licenses/by-nc/3.0/ 